\section{Different Haplotypes Encode the Same Protein for Independent Sources of Zucchini Yellow Mosaic Virus Resistance in Cucumber}

\author{
Axel O. Ramírez-Madera \\ Department of Horticulture, University of Wisconsin, Madison, WI 53706
}

Michael J. Havey ${ }^{1}$

USDA-ARS, University of Wisconsin, Madison, WI 53706

Additional index words. potyvirus, virus resistance, Zucchini yellow mosaic virus

\begin{abstract}
Cucumber (Cucumis sativus) production is negatively affected by Zucchini yellow mosaic virus (ZYMV). Three sources of ZYMV resistance have been commercially deployed and all three resistances are conditioned by a single recessive gene. A vacuolar protein sorting-associated protein 4-like (VPS4-like) gene has been proposed as a candidate for ZYMV resistance from cucumber line A192-18. We analyzed the genomic region across the VPS4-like gene for three independent sources of ZYMV resistance in cucumber (A192-18, Dina-1, and TMG-1) and identified three haplotypes across the coding region and considerable variation in the introns. However, the haplotypes in the coding regions of the VPS4-like gene of A192-18, Dina-1, and TMG1 encode the same protein sequence, revealing the genetic uniformity for ZYMV resistance from diverse germplasm sources.
\end{abstract}

The potyviruses (Potyviridae) are especially destructive for cucumber (Cucumis sativus L.) and production is often negatively affected by Watermelon mosaic virus (WMV), the watermelon strain of the Papaya ringspot virus (PRSV-W), and ZYMV. ZYMV is one of the most devastating and economically important virus diseases of cucumber worldwide (Desbiez and Lecoq, 1997; Lecoq et al., 1983; Provvidenti et al., 1984a), and several strains have been differentiated by serological and molecular approaches (Amano et al., 2013; Mahgoub et al., 1997; Nováková et al., 2014; Provvidenti et al., 1984a, 1984b). Resistance to ZYMV has been reported in cucumber. Provvidenti (1987) and Provvidenti et al. (1984a) identified a single recessive gene $\left(z^{2} m^{T M G-1}\right)$ in a selection (TMG-1) from the Chinese cucumber 'Taichung Mou Gua', which has been

Received for publication 17 Mar. 2017. Accepted for publication 29 June 2017.

This research was funded by the USDA-NIFA Specialty Crops Research Initiative grant 201151181-30661, UW Gabelman-Seminis Fellowship, and the UW Science and Medicine Graduate Research Scholars program.

We thank Christy Stewart for technical assistance in DNA extractions, Katiria González-Rivera and Erin Weber for help with sequencing and analysis software, and Doug Senalik for assembly of cucumber genomic sequences.

Disclaimer: Names are necessary to report factually on available data; however, the U.S. Department of Agriculture (USDA) neither guarantees nor warrants the standard of the product, and the use of the name by USDA implies no approval of the product to the exclusion of others that may also be suitable.

${ }^{1}$ Corresponding author. E-mail: mjhavey@wisc.edu. Gy 14.

${ }^{\mathrm{z}} \mathrm{Gy} 14$ is the reference sequence.

${ }^{\mathrm{y}}$ Resistant (R) and susceptible (S).

${ }^{\mathrm{x}}$ Sequence alignment is provided in Supplemental Fig. 1. occasionally developed a few chlorotic veins on the lower leaves. Kabelka et al. (1997) found that inoculating cotyledons of Dina-1 resulted in interveinal chlorosis on lower leaves; whereas TMG-1 had no symptoms regardless of inoculant placement. Svoboda et al. (2013) reported A192-18, G22, and TMG-1 as immune to ZYMV-H because no virus was detected by using enzyme-linked immunosorbent assay and electron microscopy.

Amano et al. (2013) proposed a VPS4-like gene as the candidate for $z y m^{A 192-18}$, which may affect virus movement throughout the plant and it is the first example of association between a vacuolar sorting protein and a recessively inherited virus resistance in plants. In this research, we analyzed sequences of the VPS4like gene from three sources of ZYMV resistance and two susceptible cucumbers, and revealed that in spite of different haplotypes, all three sources of ZYMV resistance encode the same protein sequence.

\section{Materials and Methods}

DNA was isolated from cucumber accessions ZYMV-resistant TMG-1 (Park et al., 2000; Provvidenti, 1987; Provvidenti et al., 1984a) and Dina-1 (Abul Hayja and Al-Shahwan, 1991), ZYMV-susceptible 'Straight 8' (St 8), and $49 \mathrm{~F}_{6}$ recombinant inbred lines (RILs) from a cross of TMG-1 and St 8 (Park et al., 2000) from bulked leaf tissue from at least five plants using the DNeasy ${ }^{\circledR}$ Plant Maxi Kit protocol (Qiagen, Valencia, CA). We genotyped the candidate gene for zym in St 8, TMG-1, and the 49 RILs using the cleaved amplified polymorphic (dCAPS-G99A) marker developed by Amano et al. (2013). Digested amplicons were visualized after running through $8 \%$ polyacrylamide gels and silver stained. Then, we compared the mean disease severity index scores of RILs (Park et al., 2000) carrying the resistant or susceptible genotype at the CAPS marker using a linear model in analysis of variance in RStudio (2016) version 1.0.136.

Single-end, 100 base pair (bp) sequencing runs for St 8 and TMG-1 were performed on the HiSeq2000 sequencer, version 1, SBS chemistry (Illumina, San Diego, CA). Raw reads were filtered for base quality $\geq 28$ and length $\geq 41$ nucleotides. Reads were mapped to scaffold03904 on chromosome 6 of the Gy 14 reference (Cavagnaro et al., 2010) with Bowtie2, version 2.1.0, (Langmead and Salzberg, 2012) associated protein 4-like for the Zucchini yellow mosaic locus relative to the reference sequence of

\begin{tabular}{llcc}
\hline $\begin{array}{l}\text { Haplotype } \\
\text { number }\end{array}$ & \multicolumn{1}{c}{ Line $^{\mathrm{z}}$} & $\begin{array}{c}\text { Zucchini yellow mosaic } \\
\text { virus }\end{array}$ & $\begin{array}{c}\text { Single-nucleotide } \\
\text { polymorphism variant }^{\mathrm{x}}\end{array}$ \\
\hline$\# 1$ & Gy 14 & $\mathrm{S}$ & Reference \\
$\# 2$ & St 8 & $\mathrm{~S}$ & $\mathrm{~A} 90 \mathbf{G}, \mathrm{G} 136 \mathbf{T}, \mathrm{A} 337 \mathbf{C}$ \\
$\# 3$ & TMG-1 & $\mathrm{R}$ & T86C, A90G, G99A, \\
& Dina-1 & $\mathrm{R}$ & A337C, T1150C \\
& A192-18 & $\mathrm{R}$ & T86C, A90C, \\
& & & G99A, A337C
\end{tabular}


Table 2. Three amino acid variants across the eight exons of the proposed candidate gene vacuolar protein sorting-associated protein 4-like for the Zucchini yellow mosaic locus relative to the reference sequence of Gy 14.

\begin{tabular}{llcl}
\hline $\begin{array}{l}\text { Variant } \\
\text { number }\end{array}$ & \multicolumn{1}{c}{$\begin{array}{c}\text { Zucchini yellow mosaic } \\
\text { vine }\end{array}$} & $\begin{array}{c}\text { Amino acid } \\
\text { variant }^{\mathrm{z}}\end{array}$ \\
\hline$\# 1$ & Gy 14 & $\mathrm{S}$ & Reference \\
$\# 2$ & St 8 & $\mathrm{S}$ & E46-* \\
$\# 3$ & TMG-1 & $\mathrm{R}$ & F29S, M33I \\
& Dina-1 & $\mathrm{R}$ & \\
\hline
\end{tabular}

${ }^{\mathrm{z}} \mathrm{Gy} 14$ is the reference sequence.

${ }^{\mathrm{y}}$ Resistant (R) and susceptible (S).

${ }^{\mathrm{x}}$ Amino-acid alignment is provided in Fig. 1. and converted to sorted bam format with Samtools version $0.1 .18(\mathrm{Li}, 2011)$.

Polymorphisms relative to the reference were visualized with Integrative Genome Viewer, version 2.3 (Robinson et al., 2011; Thorvaldsdottir et al., 2013). Sequences of St 8 [Sequence Read Archive (SRA) SRX1091587], TMG-1 (SRA SRX1091638), A192-18 (AB819729), and Dina-1 (KY775581) were downloaded from GenBank (https://www. ncbi.nlm.nih.gov/) and translated into amino acid (AA) sequences. Alignments of the

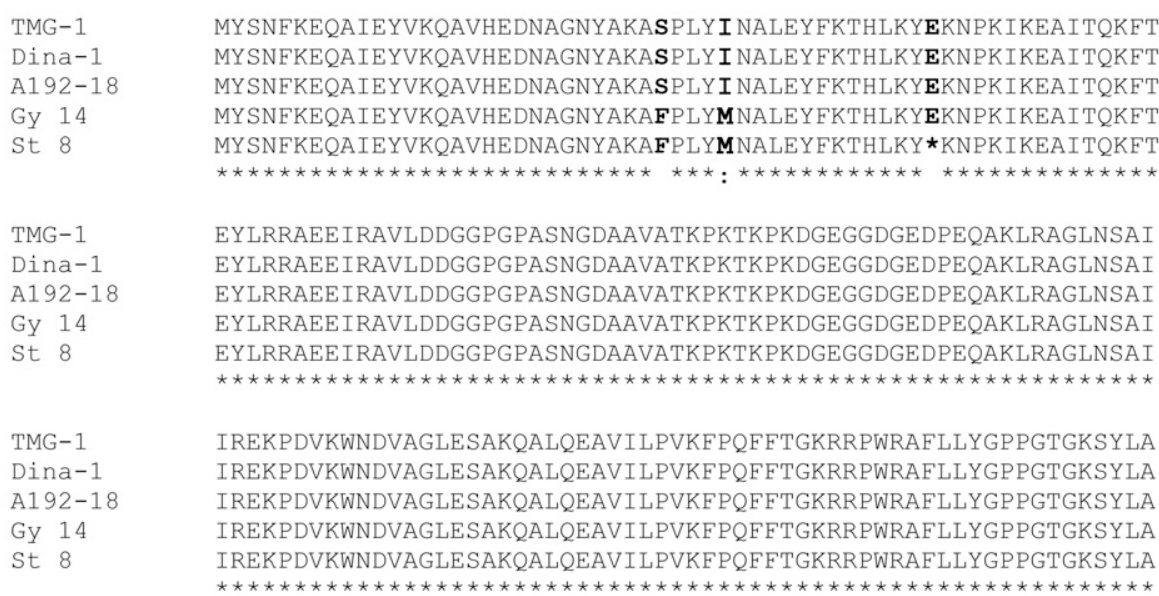

TMG-1

Dina-1

A192-18

Gy 14

St 8

TMG-1

Dina-1

A192-18

Gy 14

st 8

TMG-1

Dina-1

A192-18

Gy 14

St 8

TMG-1

Dina-1

A192-18

Gy 14

St 8

TMG-1

Dina-1

A192-18

Gy 14

St 8
KAVATEADSTFFSISSSDLVSKWMGESEKLVSNLFOMARDSAPSI IFIDEIDSLCGQRGE KAVATEADSTFFSISSSDLVSKWMGESEKLVSNLFQMARDSAPS I IFIDE IDSLCGQRGE KAVATEADSTFFSISSSDLVSKWMGESEKLVSNLFQMARDSAPSI IFIDE IDSLCGQRGE KAVATEADSTFFS ISSSDLVSKWMGESEKLVSNLFQMARDSAPS I IF I DE I DSLCGQRGE KAVATEADSTFFSISSSDLVSKWMGESEKLVSNLFQMARDSAPSIIFIDEIDSLCGQRGE

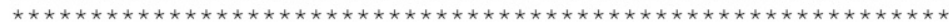

GNESEASRRIKTELLVQMQGVGHNDQKVLVLAATNTPYALDQAIRRRFDKRIYIPLPDLK GNESEASRRIKTELLVQMQGVGHNDQKVLVLAATNTPYALDQA IRRRFDKRIYIPLPDLK GNESEASRRIKTELLVQMQGVGHNDQKVLVLAATNTPYALDQAIRRRFDKRIYIPLPDLK GNESEASRRIKTELLVQMQGVGHNDQKVLVLAATNTPYALDQAIRRRFDKRIY IPLPDLK GNESEASRRIKTELLVQMQGVGHNDQKVLVLAATNTPYALDQA IRRREDKRIYIPLPDLK

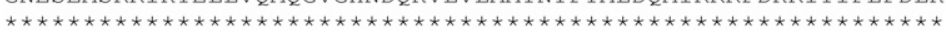

ARQHMFKVHLGDTPHNLTEADFENLARKTDGFSGSDISVCVKDVLFEPVRKTQDAMFFIM ARQHMFKVHLGDTPHNLTEADFENLARKTDGFSGSDISVCVKDVLFEPVRKTQDAMFFIM ARQHMFKVHLGDTPHNLTEADFENLARKTDGESGSDISVCVKDVLFEPVRKTQDAMFFIM ARQHMFKVHLGDTPHNLTEADFENLARKTDGFSGSDISVCVKDVLFEPVRKTQDAMFFIM ARQHMFKVHLGDTPHNLTEADFENLARKTDGFSGSDISVCVKDVLFEPVRKTQDAMFFIM

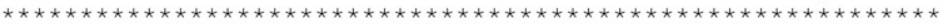

TPDGMWVPCGPKQQGAVQISMQELAAKGLASKI LPPPITRTDFDKVLARQRPTVSKSDLE TPDGMWVPCGPKQQGAVQISMQELAAKGLASKILPPP ITRTDFDKVLARQRPTVSKSDLE TPDGMWVPCGPKQQGAVQISMQELAAKGLASKILPPPITRTDFDKVLARQRPTVSKSDLE TPDGMWVPCGPKQQGAVQISMQELAAKGLASKILPPPITRTDFDKVLARQRPTVSKSDLE TPDGMWVPCGPKQQGAVQISMQELAAKGLASKILPPPITRTDEDKVLARQRPTVSKSDLE

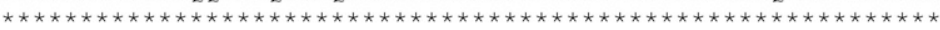

IHERFTKEFGEEG

IHERFTKEFGEEG

IHERFTKEFGEEG

IHERFTKEFGEEG

IHERFTKEFGEEG

Fig. 1. CLUSTAL O (version 1.2.4) alignment of predicted amino-acid sequences of five cucumber accessions. TMG-1, Dina-1, and A192-18 are resistant to Zucchini yellow mosaic virus, and Gy 14 and St 8 are susceptible. Polymorphisms are shown with bold text. 
coding and AA sequences of Gy 14 (Cavagnaro et al., 2010), St 8, TMG-1, A192-18, and Dina-1 were performed using CLUSTAL O, version 1.2.4 (Li et al., 2015).

The polymerase chain reaction (PCR) protocol used to amplify the $\approx 5$-kb genomic region from Dina-1 carrying the candidate gene for zym consisted of two separately prepared solutions, one which had the template DNA and the other with Taq polymerase. For a single reaction, solution 1 contained $4 \mu \mathrm{L}$ of $2.5 \mathrm{~mm}$ dNTPs, $2 \mu \mathrm{L}$ of combined forward and reverse primers (5' -TTCTTTGCTTGATTTGAGATAGTCAAAG and 5'-CAACTCTGATGGGTTGTATGCA) $(20 \mu \mathrm{m} / \mathrm{L}), 17 \mu \mathrm{L}$ of double-distilled (dd) $\mathrm{H}_{2} \mathrm{O}$, and $2 \mu \mathrm{L}$ of $20 \mathrm{ng} / \mu \mathrm{L}$ template DNA. Solution 2 had $19.25 \mu \mathrm{L}$ of dd $\mathrm{H}_{2} \mathrm{O}$, $5 \mu \mathrm{L}$ of $10 \times$ Expand High Fidelity Buffer with $15 \mathrm{mM} \mathrm{MgCl}_{2}$, and $0.75 \mu \mathrm{L}$ of Expand High Fidelity Enzyme Mix (Roche, Madison, WI). The two mixes were combined into a single tube with PCR conditions of $94^{\circ} \mathrm{C}$ for $2 \mathrm{~min}$, 30 cycles of $94^{\circ} \mathrm{C}$ for $15 \mathrm{~s}, 55^{\circ} \mathrm{C}$ for $30 \mathrm{~s}$, and $72{ }^{\circ} \mathrm{C}$ for $4.2 \mathrm{~min}$, and $72{ }^{\circ} \mathrm{C}$ for $7 \mathrm{~min}$. Amplicons were visualized in $0.6 \%$ agarose gels and remaining reaction was purified to a concentration of $50 \mathrm{ng} / \mu \mathrm{L}$ using the NucleoSpin ${ }^{\circledR}$ PCR clean-up kit (Macherey-Nagel, Düren, Germany). Amplicons were Sanger sequenced using forward primers (Supplemental Table 1) and the reverse primer listed previously by Functional Biosciences (Madison, WI), and reads were analyzed using SnapGene software version 3.3.3 (GSL Biotech, Chicago, IL).

\section{Results and Discussion}

The dCAPS-G99A marker (Amano et al., 2013) was significantly $\left(P=2.2 \times 10^{-16}\right)$ associated with reactions to ZYMV across the RILs from St $8 \times$ TMG-1 (Park et al., 2000 ), indicating that $z y m^{T M G-1}$ and $z y m^{A 192-18}$ are alleles at the same locus or tightly linked loci. Kabelka et al. (1997) previously reported that $z y m^{\text {Dina-1 }}$ and $z y m^{T M G-1}$ are also allelic or very closely linked. Together these results indicate that these three independent sources of ZYMV resistance may be one in the same.

Amano et al. (2013) reported that the candidate VPS4-like gene has eight exons and seven introns. We conducted a BLAST search using the Cucurbit Genomics Database (http://www.icugi.org/) of both the genomic and coding regions of the candidate VPS4-like gene in cucumbers 9930 and Gy 14 , and only one highly similar sequence was identified and no evidence of a duplicated gene was found. However, other gene(s) with minor effects on ZYMV resistance could exist. Further evaluation of the sequences revealed substantial variation in the VPS4like gene among the cucumber accessions Gy 14 , Dina-1, St 8, and TMG-1, with most polymorphisms in the intronic regions. Across the eight exons, six single-nucleotide polymorphisms (SNPs) define four haplotypes corresponding to Gy 14; St 8; TMG-1 and Dina-1; and A192-18 (Table 1). Across the seven introns, we identified 29 SNPs and four indels (insertions or deletions). SNPs were found in all introns (one, twelve, three, four, two, one, and six SNPs in introns one through seven, respectively). In the first intron, Dina-1 has a 37-bp duplication. In the third intron, there are four copies of a seven-bp region in Dina-1 vs. three copies in the other accessions. There are also deleted regions in intron 3, a deletion of 63-bp in Dina-1 and a 33-bp deletion at the same position in TMG-1. Intron seven carries a 3-bp deletion in Dina-1, St 8, and TMG-1 relative to Gy 14 .

The four haplotypes in the coding region for the VPS4-like gene (Table 1; Supplemental Fig. 1) encode three protein variants (Table 2; Fig. 1). The two key SNPs (T86C and G99A), which translate into two AA changes (F29S and M33I) in $z y m^{A 192-18}$ allele (Amano et al., 2013), are also present in the $z y m^{T M G-1}$ and $z y m^{\text {Dina-1 }}$ alleles (Table 2). ZYMV-susceptible Gy 14 and St 8 do not possess these two key SNPs, and St 8 has a unique SNP (G136T) that results in a premature stop codon (E46-*) (Table 2). Although A192-18, Dina-1, and TMG-1 possess different haplotypes at the VPS4-like candidate gene for zym and do not trace back to the same source population, all three haplotypes encode the same protein sequence. The most parsimonious explanation would be that the two key SNPs (T86C and G99A) occurred in a common progenitor of the original sources of ZYMV resistance introduced into Dina-1, G22, and TMG-1. Later polymorphisms may have accumulated in the coding and/or intronic regions of the gene to produce the haplotype diversity that we uncovered. Our research documents that independently identified and introgressed sources of ZYMV resistance in cucumber possess the same genetic basis and undesirable genetic uniformity exists in cucumber for potyvirus resistance that could potentially be overcome by the viruses.

\section{Literature Cited}

Abul Hayja, Z. and I.M. Al-Shahwan. 1991. Inheritance of resistance to Zucchini yellow mosaic virus in cucumber. J. Plant Dis. Prot. 98:301-304.

Al-Shahwan, I.M., O.A. Abdalla, and M.A. Al-Saleh. 1995. Response of greenhouse-grown cucumber cultivars to an isolate of Zucchini yellow mosaic virus (ZYMV). Plant Dis. 79:898-901.

Amano, M., A. Mochizuki, Y. Kawagoe, K. Iwahori, K. Niwa, J. Svoboda, T. Maeda, and Y. Imura. 2013. High-resolution mapping of zym, a recessive gene for Zucchini yellow mosaic virus resistance in cucumber. Theor. Appl. Genet. 126:2983-2993.

Cavagnaro, P.F., D.A. Senalik, L. Yang, P.W Simon, T.T. Harkins, C.D. Kodira, S. Huang, and Y. Weng. 2010. Genome-wide characterization of simple sequence repeats in cucumber (Cucumis sativus L.). BMC Genomics 11:569.

Desbiez, C. and H. Lecoq. 1997. Zucchini yellow mosaic virus. Plant Pathol. 46:809-829.

Gilbert-Albertini, F., M. Pitrat, and H. Lecoq. 1995. Inheritance of resistance to Zucchini yellow fleck virus in Cucumis sativus L. HortScience 30:336-337.

Kabelka, E. and R. Grumet. 1997. Inheritance of resistance to the Moroccan watermelon mosaic virus in the cucumber line TMG-1 and cosegregation with Zucchini yellow mosaic virus resistance. Euphytica 95:237-242.
Kabelka, E., Z. Ullah, and R. Grumet. 1997. Multiple alleles for Zucchini yellow mosaic virus resistance at the zym locus in cucumber. Theor. Appl. Genet. 95:997-1004.

Langmead, B. and S. Salzberg. 2012. Fast gappedread alignment with Bowtie 2. Nat. Methods 9:357-359.

Lecoq, H., C. Desbiez, C. Wipf-Scheibel, M. Girard, and M. Pitrat. 2002. Durability of Zucchini yellow mosaic virus resistances in cucurbits, p. 294-300. In: D.N. Maynard (ed.). Cucurbitaceae 2002. ASHS Press, Alexandria, VA.

Lecoq, H., V. Lisa, and G. Dellavalle. 1983. Serological identity of muskmelon yellow stunt and Zucchini yellow mosaic viruses. Plant Dis. $67: 824-825$

Li, H. 2011. A statistical framework for SNP calling, mutation discovery, association mapping and population genetical parameter estimation from sequencing data. Bioinformatics 27:2987-2993.

Li, W., A. Cowley, M. Uludag, T. Gur, H. McWilliam, S. Squizzato, Y.M. Park, N. Buso, and R. Lopez. 2015. The EMBL-EBI bioinformatics web and programmatic tools framework. Nucleic Acids Res. 43(W1):W580-W584.

Mahgoub, H.A., C. Desbiez, C. Wipf-Scheibel, G. Dafalla, and H. Lecoq. 1997. Characterization and occurrence of Zucchini yellow mosaic virus in Sudan. Plant Pathol. 46:800-805.

Nováková, S., J. Svoboda, and M. Glasa. 2014. Analysis of the complete sequences of two biologically distinct Zucchini yellow mosaic virus isolates further evidences the involvement of a single amino acid in the virus pathogenicity. Acta Virol. 58:364-367.

Park, Y.H., S. Sensoy, R. Antonise, J. Peleman, and M.J. Havey. 2000. A genetic map of cucumber composed of RAPDs, RFLPs, AFLPs, and loci conditioning resistance to papaya ringspot and Zucchini yellow mosaic viruses. Genome 43:1003-1010

Provvidenti, R. 1985. Sources of resistance to viruses in two accessions of Cucumis sativus. Rep. Cucurbit Genet. Coop. 8:9-12.

Provvidenti, R. 1987. Inheritance of resistance to a strain of Zucchini yellow mosaic virus in cucumber. HortScience 22:102-103.

Provvidenti, R., D. Gonsalves, and H.S. Humaydan. 1984a. Occurrence of Zucchini yellow mosaic virus in cucurbits from Connecticut, New York, Florida, and California. Plant Dis. 68:443-446.

Provvidenti, R., H.M. Munger, and H.O. Paulus. 1984b. Epidemics of Zucchini yellow mosaic virus and other cucurbit viruses in Egypt in the spring of 1983. Rep. Cucurbit Genet. Coop. 7:78-79.

Robinson, J.T., H. Thorvaldsdottir, W. Winckler, M. Guttman, E.S. Lander, G. Getz, and J.P. Mesirov. 2011. Integrative genomics viewer. Nat. Biotechnol. 29:24-26.

RStudio Team. 2016. RStudio: Integrated development for R. RStudio, Inc., Boston, MA. $<$ http://www.rstudio.com/>

Svoboda, J., L. Leisova-Svobodova, and M. Amano. 2013. Evaluation of selected cucurbitaceous vegetables for resistance to Zucchini yellow mosaic virus. Plant Dis. 97:1316-1321.

Thorvaldsdottir, H., J.T. Robinson, and J.P. Mesirov. 2013. Integrative genomics viewer (IGV): High-performance genomics data visualization and exploration. Brief. Bioinform. 14:178-192.

Ullah, Z. and R. Grumet. 2002. Localization of Zucchini yellow mosaic virus to the veinal regions and role of viral coat protein in veinal chlorosis conditioned by the zym potyvirus resistance locus in cucumber. Physiol. Mol. Plant Pathol. 60:79-89. 
Supplemental Table 1. List of primers used for sequencing of the vacuolar protein sortingassociated protein 4-like gene.

Name Forward

AR1.5 CCGGCTTCCAATGGAGATG

AR3 GAGGCTGACTCGACATTTTTCA

AR3.5 GTCGTCATTGCAATTCTTTTGTTCT

AR5 CTTGAACTTTTTACGGTTCTTGGC

AR5.5 CTCATTGCTGCTGATCTACATCC

AR7 GGGCTTAGGGGCTGTAACT

AR7.5 TCGTCACAAGTGCGTGATTC

AR9 CCATGAACTTTGCTAATATTCGTAGAT 
TMG - 1

Dina-1

A192-18

Gy 14

St 8

TMG- 1

Dina-1

A192-18

Gy 14

St 8

TMG-1

Dina-1

A192-18

Gy 14

St 8

TMG - 1

Dina-1

A192-18

Gy 14

St 8

TMG-1

Dina-1

A192-18

Gy 14

St 8

TMG - 1

Dina-1

A192-18

Gy 14

St 8

TMG-1

Dina-1

A192-18

Gy 14

st 8

TMG - 1

Dina-1

A192-18

Gy 14

St 8
ATGTATAGCAATTTCAAGGAGCAAGCTATTGAATACGTGAAGCAGGCGGTACATGAAGAT ATGTATAGCAATTTCAAGGAGCAAGCTATTGAATACGTGAAGCAGGCGGTACATGAAGAT ATGTATAGCAATTTCAAGGAGCAAGCTATTGAATACGTGAAGCAGGCGGTACATGAAGAT ATGTATAGCAATTTCAAGGAGCAAGCTATTGAATACGTGAAGCAGGCGGTACATGAAGAT ATGTATAGCAATTTCAAGGAGCAAGCTATTGAATACGTGAAGCAGGCGGTACATGAAGAT

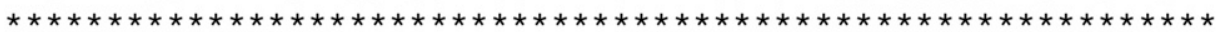

AATGCTGGCAATTACGCAAAAGCCTCTCCGCTGTATATAAACGCCTTGGAGTACTTCAAA AATGCTGGCAATTACGCAAAAGCCTCTCCGCTGTATATAAACGCCTTGGAGTACTTCAAA AATGCTGGCAATTACGCAAAAGCCTCTCCCCTGTATATAAACGCCTTGGAGTACTTCAAA AATGCTGGCAATTACGCAAAAGCCTTTCCACTGTATATGAACGCCTTGGAGTACTTCAAA AATGCTGGCAATTACGCAAAAGCCTTTCCGCTGTATATGAACGCCTTGGAGTACTTCAAA $* * * * * * * * * * * * * * * * * * * * * * * * * * * * * * * * * * * * * * * * * * * * * * * * * * * * * * * * *$

ACTCATTTGAAGTATGAGAAAAATCCCAAGATCAAGGAAGCTATTACCCAGAAATTCACC ACTCATTTGAAGTATGAGAAAAATCCCAAGATCAAGGAAGCTATTACCCAGAAATTCACC ACTCATTTGAAGTATGAGAAAAATCCCAAGATCAAGGAAGCTATTACCCAGAAATTCACC ACTCATTTGAAGTATGAGAAAAATCCCAAGATCAAGGAAGCTATTACCCAGAAATTCACC ACTCATTTGAAGTATTAGAAAAATCCCAAGATCAAGGAAGCTATTACCCAGAAATTCACC

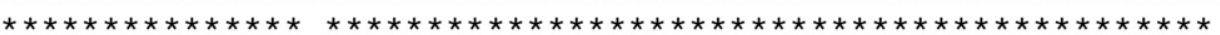

GAGTATTTGCGCCGGGCTGAGGAGATACGTGCTGTTTTGGATGATGGCGGTCCTGGTCCG GAGTATTTGCGCCGGGCTGAGGAGATACGTGCTGTTTTGGATGATGGCGGTCCTGGTCCG GAGTATTTGCGCCGGGCTGAGGAGATACGTGCTGTTTTGGATGATGGCGGTCCTGGTCCG GAGTATTTGCGCCGGGCTGAGGAGATACGTGCTGTTTTGGATGATGGCGGTCCTGGTCCG GAGTATTTGCGCCGGGCTGAGGAGATACGTGCTGTTTTGGATGATGGCGGTCCTGGTCCG

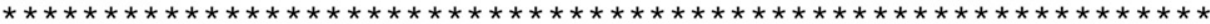

GCTTCCAATGGAGATGCAGCTGTTGCAACTAAACCCAAGACGAAACCTAAGGATGGAGAA GCTTCCAATGGAGATGCAGCTGTTGCAACTAAACCCAAGACGAAACCTAAGGATGGAGAA GCTTCCAATGGAGATGCAGCTGTTGCAACTAAACCCAAGACGAAACCTAAGGATGGAGAA GCTTCCAATGGAGATGCAGCTGTTGCAACTAAACCCAAGACGAAACCTAAGGATGGAGAA GCTTCCAATGGAGATGCAGCTGTTGCAACTAAACCCAAGACGAAACCTAAGGATGGAGAA

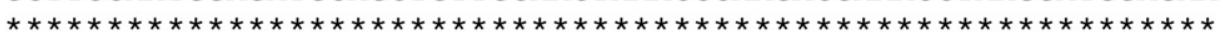

GGAGGAGATGGAGAGGATCCAGAACAGGCTAAGTTACGGGCTGGTCTTAATTCCGCAATC GGAGGAGATGGAGAGGATCCAGAACAGGCTAAGTTACGGGCTGGTCTTAATTCCGCAATC GGAGGAGATGGAGAGGATCCAGAACAGGCTAAGTTACGGGCTGGTCTTAATTCCGCAATC GGAGGAGATGGAGAGGATCCAGAACAGGCTAAGTTAAGGGCTGGTCTTAATTCCGCAATC GGAGGAGATGGAGAGGATCCAGAACAGGCTAAGTTACGGGCTGGTCTTAATTCCGCAATC $* * * * * * * * * * * * * * * * * * * * * * * * * * * * * * * * * * * * * * * * * * * * * * * * * * * * * * * * * * * *$

ATAAGGGAGAAACCGGATGTTAAGTGGAACGATGTTGCTGGATTGGAGAGCGCCAAGCAG ATAAGGGAGAAACCGGATGTTAAGTGGAACGATGTTGCTGGATTGGAGAGCGCCAAGCAG ATAAGGGAGAAACCGGATGTTAAGTGGAACGATGTTGCTGGATTGGAGAGCGCCAAGCAG ATAAGGGAGAAACCGGATGTTAAGTGGAACGATGTTGCTGGATTGGAGAGCGCCAAGCAG ATAAGGGAGAAACCGGATGTTAAGTGGAACGATGTTGCTGGATTGGAGAGCGCCAAGCAG

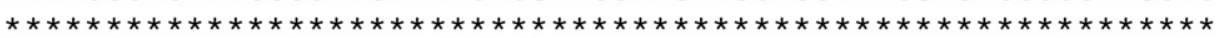

GCGTTGCAAGAGGCTGTAATATTACCCGTTAAGTTCCCCCAGTTCTTTACTGGTAAGAGG GCGTTGCAAGAGGCTGTAATATTACCCGTTAAGTTCCCCCAGTTCTTTACTGGTAAGAGG GCGTTGCAAGAGGCTGTAATATTACCCGTTAAGTTCCCCCAGTTCTTTACTGGTAAGAGG GCGTTGCAAGAGGCTGTAATATTACCCGTTAAGTTCCCCCAGTTCTTTACTGGTAAGAGG GCGTTGCAAGAGGCTGTAATATTACCCGTTAAGTTCCCCCAGTTCTTTACTGGTAAGAGG

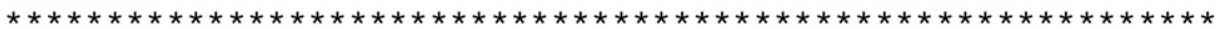

Supplemental Fig. 1. CLUSTAL O (version 1.2.4) alignment of nucleotide coding sequences of five cucumber accessions. TMG-1, Dina-1, and A192-18 are resistant to Zucchini yellow mosaic virus, and Gy 14 and St 8 are susceptible. Polymorphisms are shown with bold text. 
TMG - 1

Dina-1

A192-18

Gy 14

St 8

TMG - 1

Dina-1

A192-18

Gy 14

St 8

TMG - 1

Dina-1

A192-18

Gy 14

St 8

TMG - 1

Dina-1

A192-18

Gy 14

St 8

TMG - 1

Dina-1

A192-18

Gy 14

st 8

TMG - 1

Dina-1

A192-18

Gy 14

St 8

TMG - 1

Dina-1

A192-18

Gy 14

St 8

TMG - 1

Dina-1

A192-18

Gy 14

st 8
CGACCATGGCGAGCTTTCTTGTTATATGGGCCTCCTGGAACTGGAAAGTCATACTTGGCA CGACCATGGCGAGCTTTCTTGTTATATGGGCCTCCTGGAACTGGAAAGTCATACTTGGCA CGACCATGGCGAGCTTTCTTGTTATATGGGCCTCCTGGAACTGGAAAGTCATACTTGGCA CGACCATGGCGAGCTTTCTTGTTATATGGGCCTCCTGGAACTGGAAAGTCATACTTGGCA CGACCATGGCGAGCTTTCTTGTTATATGGGCCTCCTGGAACTGGAAAGTCATACTTGGCA

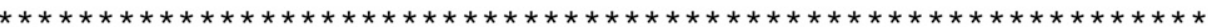

AAGGCTGTTGCGACAGAGGCTGACTCGACATTTTTCAGCATTTCTTCTTCGGACCTTGTC AAGGCTGTTGCGACAGAGGCTGACTCGACATTTTTCAGCATTTCTTCTTCGGACCTTGTC AAGGCTGTTGCGACAGAGGCTGACTCGACATTTTTCAGCATTTCTTCTTCGGACCTTGTC AAGGCTGTTGCGACAGAGGCTGACTCGACATTTTTCAGCATTTCTTCTTCGGACCTTGTC AAGGCTGTTGCGACAGAGGCTGACTCGACATTTTTCAGCATTTCTTCTTCGGACCTTGTC $\star * * * * * * * * * * * * * * * * * * * * * * * * * * * * * * * * * * * * * * * * * * * * * * * * * * * * * * * * * * *$

TCGAAATGGATGGGTGAAAGCGAGAAGCTAGTTTCAAATCTTTTTCAAATGGCTCGTGAT TCGAAATGGATGGGTGAAAGCGAGAAGCTAGTTTCAAATCTTTTTCAAATGGCTCGTGAT TCGAAATGGATGGGTGAAAGCGAGAAGCTAGTTTCAAATCTTTTTCAAATGGCTCGTGAT TCGAAATGGATGGGTGAAAGCGAGAAGCTAGTTTCAAATCTTTTTCAAATGGCTCGTGAT TCGAAATGGATGGGTGAAAGCGAGAAGCTAGTTTCAAATCTTTTTCAAATGGCTCGTGAT

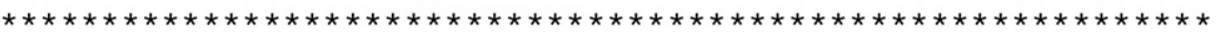

AGCGCGCCTTCTATCATCTTTATTGATGAAATAGATTCGTTGTGTGGTCAACGAGGTGAA AGCGCGCCTTCTATCATCTTTATTGATGAAATAGATTCGTTGTGTGGTCAACGAGGTGAA AGCGCGCCTTCTATCATCTTTATTGATGAAATAGATTCGTTGTGTGGTCAACGAGGTGAA AGCGCGCCTTCTATCATCTTTATTGATGAAATAGATTCGTTGTGTGGTCAACGAGGTGAA AGCGCGCCTTCTATCATCTTTATTGATGAAATAGATTCGTTGTGTGGTCAACGAGGTGAA $\star * * * * * * * * * * * * * * * * * * * * * * * * * * * * * * * * * * * * * * * * * * * * * * * * * * * * * * * * * * *$

GGTAATGAAAGTGAAGCTTCAAGACGCATTAAGACTGAACTTCTTGTGCAAATGCAGGGT GGTAATGAAAGTGAAGCTTCAAGACGCATTAAGACTGAACTTCTTGTGCAAATGCAGGGT GGTAATGAAAGTGAAGCTTCAAGACGCATTAAGACTGAACTTCTTGTGCAAATGCAGGGT GGTAATGAAAGTGAAGCTTCAAGACGCATTAAGACTGAACTTCTTGTGCAAATGCAGGGT GGTAATGAAAGTGAAGCTTCAAGACGCATTAAGACTGAACTTCTTGTGCAAATGCAGGGT

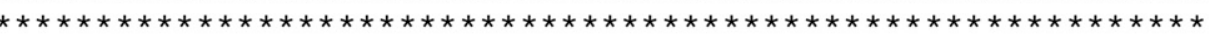

GTAGGACACAATGATCAAAAGGTTCTTGTTCTTGCTGCAACAАATACTCCCTATGCTTTG GTAGGACACAATGATCAAAAGGTTCTTGTTCTTGCTGCAACAAATACTCCCTATGCTTTG GTAGGACACAATGATCAAAAGGTTCTTGTTCTTGCTGCAACAAATACTCCCTATGCTTTG GTAGGACACAATGATCAAAAGGTTCTTGTTCTTGCTGCAACAAATACTCCCTATGCTTTG GTAGGACACAATGATCAAAAGGTTCTTGTTCTTGCTGCAACAAATACTCCCTATGCTTTG $* * * * * * * * * * * * * * * * * * * * * * * * * * * * * * * * * * * * * * * * * * * * * * * * * * * * * * * * * * * *$

GATCAGGCCATTCGTCGACGATTCGACAAGCGGATATACATTCCTCTACCTGATTTGAAA GATCAGGCCATTCGTCGACGATTCGACAAGCGGATATACATTCCTCTACCTGATTTGAAA GATCAGGCCATTCGTCGACGATTCGACAAGCGGATATACATTCCTCTACCTGATTTGAAA GATCAGGCCATTCGTCGACGATTCGACAAGCGGATATACATTCCTCTACCTGATTTGAAA GATCAGGCCATTCGTCGACGATTCGACAAGCGGATATACATTCCTCTACCTGATTTGAAA

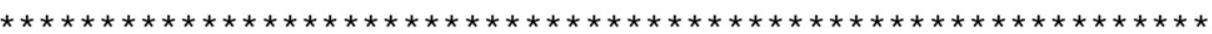

GCCAGACAGCACATGTTCAAAGTGCATCTGGGGGATACACCACATAATTTAACAGAAGCA GCCAGACAGCACATGTTCAAAGTGCATCTGGGGGATACACCACATAATTTAACAGAAGCA GCCAGACAGCACATGTTCAAAGTGCATCTGGGGGATACACCACATAATTTAACAGAAGCA GCCAGACAGCACATGTTCAAAGTGCATCTGGGGGATACACCACATAATTTAACAGAAGCA GCCAGACAGCACATGTTCAAAGTGCATCTGGGGGATACACCACATAATTTAACAGAAGCA

Fig. 1. continued 
TMG - 1

Dina-1

A192-18

Gy 14

st 8

TMG - 1

Dina-1

A192-18

Gy 14

st 8

TMG - 1

Dina-1

A192-18

Gy 14

St 8

TMG - 1

Dina-1

A192-18

Gy 14

St 8

TMG - 1

Dina-1

A192-18

Gy 14

st 8

TMG - 1

Dina-1

A192-18

Gy 14

St 8
GATTTTGAAAACTTAGCACGCAAGACAGATGGTTTCTCGGGTTCAGATATTTCAGTTTGT GATTTTGAAAACTTAGCACGCAAGACAGATGGTTTCTCGGGTTCAGATATTTCAGTTTGT GATTTTGAAAACTTAGCACGCAAGACAGATGGTTTCTCGGGTTCAGATATTTCAGTTTGT GATTTTGAAAACTTAGCACGCAAGACAGATGGTTTCTCGGGTTCAGATATTTCAGTTTGT GATTTTGAAAACTTAGCACGCAAGACAGATGGTTTCTCGGGTTCAGATATTTCAGTTTGT



GTGAAGGATGTGCTCTTTGAACCTGTACGTAAAACTCAAGATGCTATGTTCTTCATTATG GTGAAGGATGTGCTCTTTGAACCTGTACGTAAAACTCAAGATGCTATGTTCTTCATTATG GTGAAGGATGTGCTCTTTGAACCTGTACGTAAAACTCAAGATGCTATGTTCTTCATTATG GTGAAGGATGTGCTCTTTGAACCTGTACGTAAAACTCAAGATGCTATGTTCTTCATTATG GTGAAGGATGTGCTCTTTGAACCTGTACGTAAAACTCAAGATGCTATGTTCTTCATTATG

$* * * * * * * * * * * * * * * * * * * * * * * * * * * * * * * * * * * * * * * * * * * * * * * * * * * * * * * * * * * *$

ACTCCTGATGGTATGTGGGTACCTTGTGGACCAAAGCAACAAGGAGCTGTCCAAATTAGC ACTCCTGATGGTATGTGGGTACCTTGTGGACCAAAGCAACAAGGAGCTGTCCAAATTAGC ACTCCTGATGGTATGTGGGTACCTTGTGGACCAAAGCAACAAGGAGCTGTCCAAATTAGC ACTCCTGATGGTATGTGGGTACCTTGTGGACCAAAGCAACAAGGAGCTGTCCAAATTAGC ACTCCTGATGGTATGTGGGTACCTTGTGGACCAAAGCAACAAGGAGCTGTCCAAATTAGC

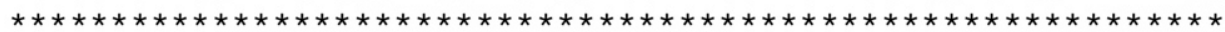

ATGCAAGAGCTAGCAGCCAAAGGACTTGCCTCAAAGATTCTTCCTCCACCAATTACAAGA ATGCAAGAGCTAGCAGCCAAAGGACTTGCCTCAAAGATTCTTCCTCCACCAATTACAAGA ATGCAAGAGTTAGCAGCCAAAGGACTTGCCTCAAAGATTCTTCCTCCACCAATTACAAGA ATGCAAGAGTTAGCAGCCAAAGGACTTGCCTCAAAGATTCTTCCTCCACCAATTACAAGA ATGCAAGAGTTAGCAGCCAAAGGACTTGCCTCAAAGATTCTTCCTCCACCAATTACAAGA

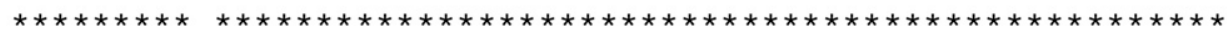

ACAGATTTTGACAAGGTCTTAGCTAGACAAAGGCCTACGGTGAGTAAATCTGATCTGGAA ACAGATTTTGACAAGGTCTTAGCTAGACAAAGGCCTACGGTGAGTAAATCTGATCTGGAA ACAGATTTTGACAAGGTCTTAGCTAGACAAAGGCCTACGGTGAGTAAATCTGATCTGGAA ACAGATTTTGACAAGGTCTTAGCTAGACAAAGGCCTACGGTGAGTAAATCTGATCTGGAA ACAGATTTTGACAAGGTCTTAGCTAGACAAAGGCCTACGGTGAGTAAATCTGATCTGGAA

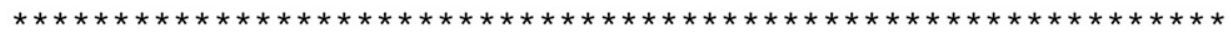

ATTCATGAAAGGTTTACAAAAGAGTTTGGGGAGGAAGGTTGA ATTCATGAAAGGTTTACAAAAGAGTTTGGGGAGGAAGGTTGA ATTCATGAAAGGTTTACAAAAGAGTTTGGGGAGGAAGGTTGA ATTCATGAAAGGTTTACAAAAGAGTTTGGGGAGGAAGGTTGA ATTCATGAAAGGTTTACAAAAGAGTTTGGGGAGGAGGTTGA

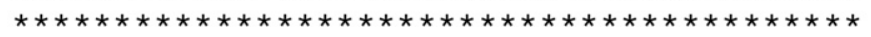

Fig. 1. continued 